\section{Packet delay analysis of IEEE 802.11 MAC protocol}

\section{P. Chatzimisios, A.C. Boucouvalas and V. Vitsas}

A new packet delay analysis for the IEEE $802.11 \mathrm{~b}$ protocol is developed by extending throughput analysis introduced by Bianchi. This analysis is validated by comparison with simulation results using the $\mathrm{OPNET}^{\mathrm{TM}}$ simulation package. Packet delay results are presented as a function of a number of stations and packet size for basic access and RTS/CTS medium access mechanisms. The proposed analysis identifies when the RTS/CTS mechanism achieves lower packet delay with respect to the basic access mechanism and becomes highly beneficial for the performance of IEEE 802.11 WLANs.

Introduction: Wireless local area networks (WLANs) are one of the fastest growing areas in data communications and networking, and have witnessed a tremendous development over the past few years [1]. The IEEE working group proposed the 802.11 protocol [2] to deal with the modern wireless connectivity needs. The IEEE 802.11 protocol is a carrier sense multiple access with collision avoidance (CSMA/CA) MAC protocol using binary exponential backoff. The fundamental technique to access the medium is called distributed coordination function (DCF). DCF defines two access mechanisms to employ packet transmission, the basic access and the Request-To-Send/ Clear-To-Send (RTS/CTS). A station with a packet to transmit senses the medium activity. If the medium is idle, the station transmits. If the medium is sensed busy, the station persists to monitor the medium until it becomes idle for more than distributed inter-frame space (DIFS) time period. To minimise collisions the station waits a random backoff interval before transmitting the data packet. If the collision probability is high and the packet size is longer than a threshold, the RTS/CTS reservation scheme is used. In this case, short RTS and CTS packets are exchanged to reserve the medium prior to the transmission of the long data packet. The RTS/CTS scheme shortens collision duration and copes with hidden stations.

In this Letter a mathematical analysis is developed that calculates the average packet delay for both medium access mechanisms. Our analysis extends the approach in $[3,4]$ by utilising the same Markov chain model for calculating the average packet delay. We present results, which specify the threshold packet size above which the use of the RTS/CTS mechanism is superior to the basic access. As the RTS/CTS efficiency depends on the packet collision probability, which increases for large networks, we evaluate the dependency of the RTS/CTS threshold on network size. Furthermore, we identify the network and traffic conditions where the RTS/CTS mechanism enhances the performance of the IEEE 802.11 MAC protocol.

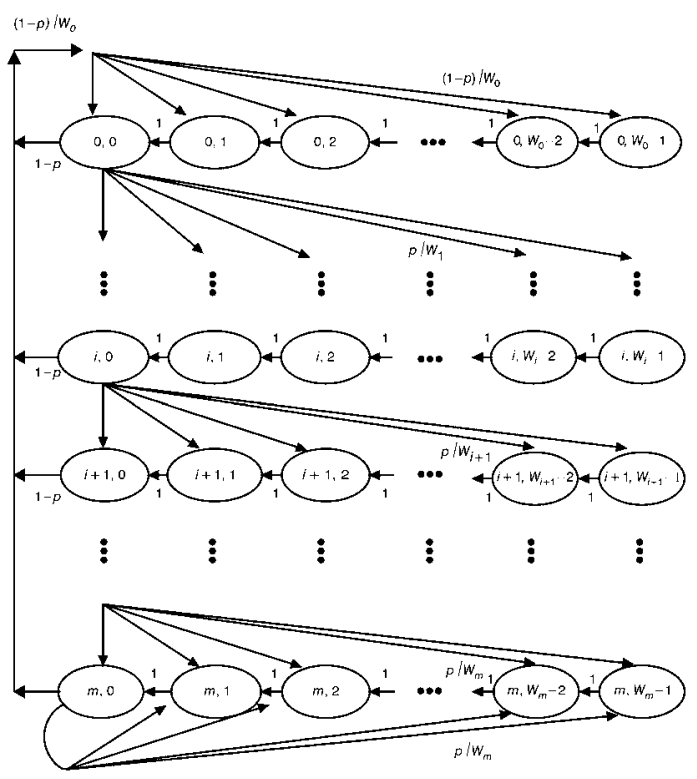

Fig. 1 Markov chain model for backoff $C W$
Analysis: We assume that: (i) the network consists of $n$ contending stations, (ii) each station always has a packet available for transmission and (iii) the collision probability $p$ of a transmitted packet is constant and independent of the retransmissions this packet has suffered in the past. Prior to initiating a packet transmission, the value of the station's backoff timer is uniformly chosen in the range $\left[0, W_{i}-1\right]$, where $W_{i}$ is the current contention window (CW) size and $i$ is the backoff stage. We have $W_{i}=2^{i} \cdot W, i \in[0, m]$, where $W$ is the minimum $\mathrm{CW}$ size and $m$ is the maximum backoff stage such that $\mathrm{CW}_{\max }=2^{m} \cdot W$. At the first transmission attempt of a packet, $W=$ $\mathrm{CW}_{\min }=W_{o}$ and after an unsuccessful transmission, $W_{i}$ is doubled up to the maximum value $\mathrm{CW}_{\max }$. According to IEEE $802.11 \mathrm{~b}$ standard [2], we have $\mathrm{CW}_{\min }=32, \mathrm{CW}_{\max }=1024$ and $m=5$. Let $b(t)$ be the backoff timer and $s(t)$ the backoff stage for a given station at time $t$. The discrete-time Markov chain shown in Fig. 1 is employed to model the bi-dimensional process $\{b(t), s(t)\}$. Let $b_{i, k}=\lim _{t \rightarrow \infty} P\{s(t)=i$, $b(t)=k\}$ be the stationary distribution of the Markov chain, where $i \in[0, m], k \in\left[0, W_{i}-1\right]$. Using the Markov chain, the probability $\tau$ that a station transmits a packet in a randomly chosen slot time $[3,4]$ is equal to:

$$
\tau=\frac{2 \cdot(1-2 p)}{(1-2 p) \cdot(W+1)+p W \cdot\left(1-(2 p)^{m}\right)}
$$

and the probability $p$ that a transmitted packet encounters a collision is given by:

$$
p=1-(1-\tau)^{n-1}
$$

(1) and (2) form a nonlinear system with two unknowns $\tau$ and $p$ which can be solved using numerical methods.

Throughput analysis in [3] and [4] calculates the saturation throughput $S$ which is the maximum load that the system can carry in stable conditions. $S$ can be found by dividing the transmitted payload information in a slot time with the average length of a slot time:

$$
\begin{aligned}
S & =\frac{P_{t r} \cdot P_{s} \cdot l}{E[\text { slot }]} \\
& =\frac{P_{t r} \cdot P_{s} \cdot l}{\left(1-P_{t r}\right) \cdot \sigma+P_{t r} \cdot P_{s} \cdot T_{s}+P_{t r} \cdot\left(1-P_{s}\right) \cdot T_{c}}
\end{aligned}
$$

where $E[$ slot $]$ is the average length of a slot time, $\sigma$ is the duration of an empty slot time, $P_{t r}$ is the probability that there is at least one packet transmission in a randomly chosen slot time, $P_{s}$ the probability that an occurring packet transmission is successful, $T_{c}$ and $T_{s}$ are the average durations the medium is sensed busy due to a collision and a successful transmission, respectively. The probabilities $P_{t r}$ and $P_{s}$ are given by:

$$
P_{t r}=1-(1-\tau)^{n}, \quad P_{s}=\frac{n \cdot \tau \cdot(1-\tau)^{n-1}}{1-(1-\tau)^{n}}
$$

Our analysis calculates the average delay $E[D]$ for a successfully transmitted packet. Packet delay is defined to be the time interval from the time a packet is at the head of its MAC queue ready for transmission, until its successful reception in the destination. $E[D]$ is given by:

$$
E[D]=E[X] \cdot E[\text { slot }]
$$

where $E[X]$ is the average number of slot times for a successful packet transmission. $E[X]$ can be found by multiplying the number of slot times $d_{i}$ the packet is delayed in each backoff stage by the probability $q_{i}$ to reach this backoff stage. More specifically:

$$
\begin{aligned}
& E[X]=d_{i} \cdot q_{i} \\
& d_{i}=\frac{W_{i}+1}{2}, \quad i \in[0, m] \\
& q_{i}= \begin{cases}p^{i}, & i \in[0, m-1] \\
\frac{p^{m}}{1-p}, & i=m\end{cases}
\end{aligned}
$$

After some algebra, $E[X]$ is given by:

$$
E[X]=\frac{(1-2 p) \cdot(W+1)+p W \cdot\left(1-(2 p)^{m}\right)}{2 \cdot(1-2 p) \cdot(1-p)}
$$

Results: Fig. 2 shows the average packet delay and throughput efficiency against the number of contenting stations for packets of 
size $l=8184$ bits. The Figure validates our mathematical analysis since an almost exact match is observed between analytical (symbols) and simulation results (lines) from our IEEE 802.11 simulator developed with the OPNET ${ }^{\mathrm{TM}}$ simulation package. The Figure shows that the RTS/CTS mechanism achieves lower delay and higher throughput values than the basic access mechanism, for the specific large packet size, as a result of the shorter duration of the collisions.

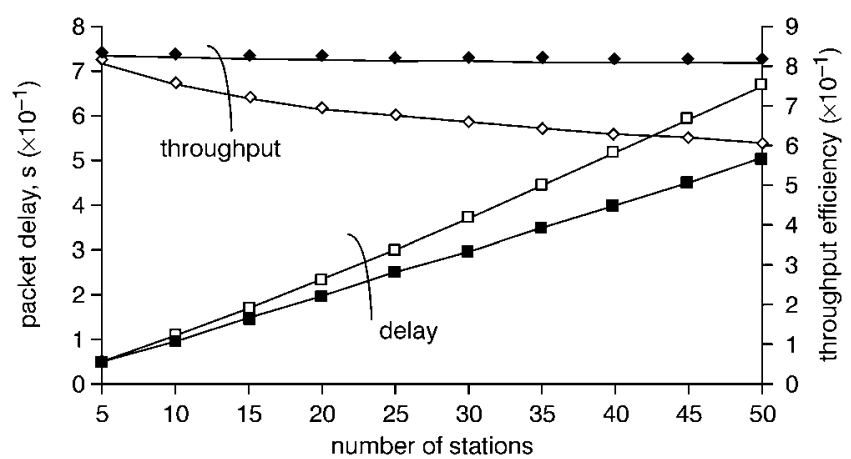

Fig. 2 Packet delay and throughput efficiency against $n$, for $W=32$, $l=8184$ bits, $m=5, C=1 \mathrm{Mbit} / \mathrm{s}$

$\diamond$ throughput efficiency, RTS/CTS

$\diamond$ throughput efficiency, basic access

- packet delay, RTS/CTS

packet delay, basic access

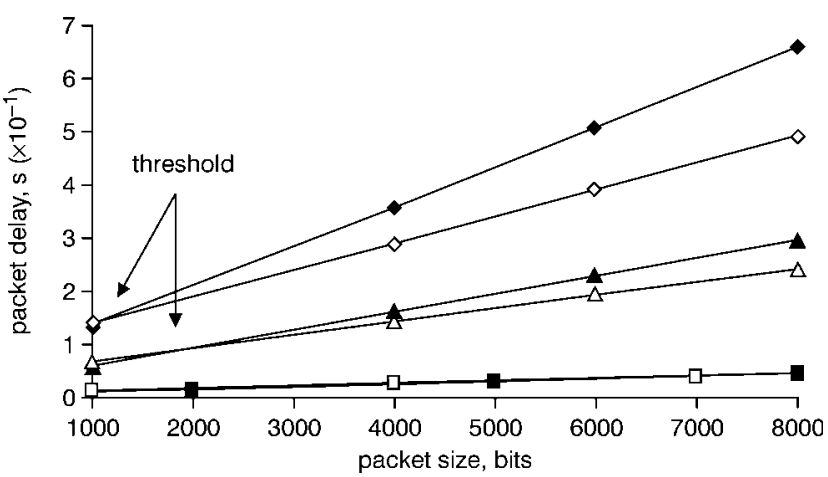

Fig. 3 Packet delay against $l$ for various $n$ values, $W=32, \quad m=5$, $C=1 \mathrm{Mbit} / \mathrm{s}$
$\bullet$ basic access, $n=50$
- basic access, $n=5$
$\Delta$ basic access, $n=25$
$\diamond$ RTS $/$ CTS,$n=50$
$\triangle \mathrm{RTS} / \mathrm{CTS}, n=25$
$\square$ RTS/CTS, $n=5$

Fig. 3 shows the average packet delay against packet size for both the basic access and RTS/CTS cases, for three representative network sizes
( $n=5,25$ and 50). The Figure examines the threshold value above which the performance of the RTS/CTS mechanism is considerably enhanced. If the number of the contending stations is relatively small $(n=5)$, the basic access achieves equivalent packet delay compared to the RTS/CTS mechanism, for all packet size values. This shows that the RTS/CTS mechanism is not advantageous in small size networks due to the low collision probability. On the contrary, when the network size $n$ increases to 25 stations and the packet size exceeds a specific threshold (1900 bits), the RTS/CTS mechanism achieves a lower packet delay value compared to the basic access. This threshold decreases to about 1300 bits for large network scenarios $(n=50)$. In this case, the RTS/CTS mechanism is particularly beneficial due to the increase of the collision probability.

Conclusions: This Letter proposes a new analytical model to compute the average packet delay of the IEEE 802.11 protocol. This analysis is validated by comparison with simulation results. Protocol performance results indicate that the RTS/CTS mechanism does not result in significant packet delay improvement for small networks. However, in large network scenarios, the RTS/CTS mechanism is extremely beneficial with respect to the basic access mechanism, achieving a lower packet delay value especially when long packets are transmitted. These results are essential in determining the optimal use of the RTS/CTS mechanism that significantly improves the performance of IEEE 802.11 WLANs.

(C) IEE 2003

Electronics Letters Online No: 20030868

16 May 2003 DOI: 10.1049/el:20030868

P. Chatzimisios and A.C. Boucouvalas (Design, Engineering and Computing, Bournemouth University, Fern Barrow, Poole, Dorset, BH12 5BB, United Kingdom)

V. Vitsas (Department of Information Technology, Technological Educational Institution, Thessaloniki, Greece)

\section{References}

1 NEE, R., and AWATER, G.: 'New high-rate wireless LAN standards', IEEE Commun. Mag., 1999, 37, (12), pp. 82-88

2 IEEE Standard for Wireless LAN Medium Access Control (MAC) and Physical Layer (PHY) specifications, IEEE Std: 802.11b-1999/Cor 1-2001, 2001

3 BIANCHI, G.: 'IEEE 802.11: saturation throughput analysis', IEEE Commun. Lett., 1998, 2, (12), pp. 318-320

4 BIANCHI, G.: 'Performance analysis of the IEEE 802.11 distributed coordination function', IEEE J. Sel. Areas Commun., 2000, 18, (3), pp. $535-547$ 